\title{
Towards secure model for SCADA systems
}

\begin{abstract}
Nowadays, Supervisory Control And Data Acquisition (SCADA) systems have huge influences on the human life. They provide a distance remote controlling, monitoring and information gathering of transmission, production and distribution of every automation systems such as electric power, power plants, refineries, rail transportation, waste and water systems, oil and gas. In this paper, different possible threats, risks and vulnerabilities in the SCADA systems are surveyed, and some mitigation strategies to improve the SCADA security systems are proposed.
\end{abstract}

Keyword: SCADA security; SCADA threats; SCADA vulnerabilities; SCADA security preventions 